\title{
Engineering Fe-N doped Graphene to mimic biological functions of NADPH oxidase in cells
}

Di Wu ${ }^{\dagger \#}$, Jingkun $\mathrm{Li}^{\ddagger}{ }^{\ddagger}$, Shujuan $\mathrm{Xu}^{\dagger}$, Qianqian $\mathrm{Xie}^{\dagger}$, Yanxia Pan ${ }^{\dagger}$, Xi Liu ${ }^{\dagger}$, Ronglin $\mathrm{Ma}^{\dagger}$, Huizhen Zheng ${ }^{\dagger}$, Meng $\mathrm{Gao}^{\dagger}$, Weili Wang ${ }^{\dagger}$, Jia Li ${ }^{\dagger}$, Xiaoming Cai ${ }^{\dagger}$, Frédéric Jaouen ${ }^{\S *}$, Ruibin $\mathrm{Li}^{\dagger *}$

†State Key Laboratory of Radiation Medicine and Protection, School for Radiological and interdisciplinary Sciences (RAD-X), Collaborative innovation Center of radiological Medicine of Jiangsu Higher Education Institutions, Soochow University, Suzhou 215123, Jiangsu China

Key Laboratory for Green Chemical Technology of Ministry of Education, School of Chemical Engineering and Technology, Tianjin University; Collaborative Innovation Center of Chemical Science and Engineering (Tianjin), Tianjin 300072, China

$\S$ ICGM, Univ. Montpellier, CNRS, ENSCM, Montpellier, France

\# equal contribution

*Address correspondence to

Dr. Frédéric Jaouen

$\&$

Dr. Ruibin Li

Email: frederic.jaouen@umontpellier.fr

Email: liruibin@suda.edu.cn

Tel: $+33-467143211$

Tel: $+86-512-65880062$ 


\begin{abstract}
NADPH oxidase (NOX) as a transmembrane protein complex controls the generation of superoxide that plays important roles in immune signaling pathway. NOX inactivation may elicit immunodeficiency and cause chronic granulomatous disease (CGD). Biocompatible synthetic materials with NOX-like activities would therefore be interesting as curative and/or preventive approaches in case of NOX deficiency. Herein, we synthesized a Fe-N doped graphene (FeNGR) nanomaterial that could mimic the activity of NOX by efficiently catalyzing the conversion of NADPH into NADP ${ }^{+}$and triggering the generation of oxygen radicals. The resulting FeNGR nanozyme had similar cellular distribution to NOX, and is able to mimic the enzyme function in NOXdeficient cells by catalyzing the generation of superoxide and retrieving the immune activity, evidenced by IL-1 $\beta$ and TNF- $\alpha$ production in response to Alum exposure. Overall, our study discovered the first synthetic materials to mimic NOX and demonstrated the enzyme function of FeNGR nanozyme in cells.
\end{abstract}

Key words: Nanocatalyst, NADPH oxidase, Nanozyme, Graphene, Doping 


\section{Introduction}

The membrane-bound oxidase enzyme called nicotinamide adenine dinucleotide phosphate (NADPH) oxidase is crucial to maintain a healthy level of reactive oxygen species (ROS) in phagocytic and vascular cells of mammals ${ }^{[1]}{ }^{[2]}$. NADPH oxidase (NOX) consists of five subunits: three cytosolic subunits ( $\mathrm{p} 40^{\text {phox }}, \mathrm{p} 47^{\text {phox }}$ and $\left.\mathrm{p} 67^{\text {phox }}\right)$, a cytochrome b558 (gp91 ${ }^{\text {phox }}$, p22 ${ }^{\text {phox }}$ ) and a small G protein Rac ${ }^{[3]}$. Cytosolic subunits and gp91 $91^{\text {phox }}$ take part in the hydrogen abstraction of NADPH and one-electron reduction of molecular oxygen, respectively. Small G protein is a crucial switch to control the activation of the oxidase. While this enzyme is dormant under normal circumstances, it is activated during respiratory burst to generate superoxide and activate immune responses against foreign stimuli ${ }^{[4]}$. NOX deficiency may directly cause chronic granulomatous disease (CGD) in which the immune cells fail to generate essential oxygen radicals and burst immune responses for clearance of pathogenic bacteria and fungi ${ }^{[5]}$. Thus, patients with CGD experience recurrent infections and are also easy to develop severe granulomas resulting in obstructive lesions in the esophagus, stomach, and urinary tract ${ }^{[6]}$. Although NOX is crucial to the normal functioning of the immune system of cells ${ }^{[7]}{ }^{[8]}$, the purification and reconstruction of this enzyme is very challenging. In addition, while being attractive and logical, no attempts have been made hitherto to explore synthetic materials for mimicking the enzymatic activity of NOX.

Toward this goal, the rapid development of nanotechnologies has provided opportunities to engineer inorganic and/or organic nanocatalysts with enzyme-like activity, a.k.a. nanozymes ${ }^{[9]}$. For instance, $\mathrm{Fe}_{3} \mathrm{O}_{4}$ and graphene oxide were found to display peroxidase-like activity ${ }^{[10]}{ }^{[11]}$; carbon nitride and gold nanoparticles showed oxidase-like activity ${ }^{[12]}$; noble metal nanoparticles and $\mathrm{SnSe}$ nanosheets exhibited superoxide dismutase- ${ }^{[13]}$ and dehydrogenase-like ${ }^{[14]}$ activities, respectively. While 
these nanozymes have been reported to catalyze the degradation of $\mathrm{H}_{2} \mathrm{O}_{2}, \mathrm{O}_{2}{ }^{\circ-}$ and hydrogen transfer from 1-(R)-2-(R')-ethanol group, there is yet no identified nanozyme to mimic a complex enzyme, like NOX for catalyzing the formation of superoxide radicals via selective hydrogen abstraction and one-electron transfer from NADPH to oxygen. Considering the decisive role of the heme core of NOX in the electron transfer from NADPH, we hypothesized that Fe-N doped graphene (FeNGR) nanosheets with embedded single-metal atom catalytic sites with heme-like local coordination are able to mimic the biological functions of NOX in cells (Figure S1).

In this study, Fe- $\mathrm{N}_{\mathrm{x}}$ moieties covalently embedded in graphene (GR) sheets were prepared and characterized. We exploited liquid chromatography to examine the conversion of NADPH into $\mathrm{NADP}^{+}$and a radical-sensitive fluorescent substrate to detect the generation of oxygen radicals. The enzymatic performance of FeNGR nanozyme was examined in different reaction conditions for a comparison to protein enzyme. Finally, we assessed the capability of FeNGR to mimic the biological functions of NOX in a macrophage-like THP-1 cells by detection of superoxide generation and immune cytokine release.

\section{Results}

\section{Synthesis and characterization of Fe-N doped graphene}

FeNGR was prepared by pyrolysis of well-mixed iron acetate, 1,10-phenanthroline and ZIF-8 at $1050{ }^{\circ} \mathrm{C}$ according to a previously reported method ${ }^{[15]}$. The resulting materials were characterized by atomic force microscopy (AFM), Raman spectroscopy, extended X-ray absorption fine structure (EXAFS) and ${ }^{57} \mathrm{Fe}$ Mössbauer spectroscopy. The AFM images showed that FeNGR had irregular planar structure with 300-400 nm diameters, and few atomic monolayers with height of 1.2-1.5 nm, similar to pristine GR (Figure 1A). Typical D and G bands of GR lattice could be detected with Raman spectroscopy at 1328 and $1600 \mathrm{~cm}^{-1}$, respectively (Figure 1B). We then resorted to EXAFS and ${ }^{57} \mathrm{Fe}$ Mössbauer spectroscopy to identify the local structure and coordination environment of iron in FeNGR. The Fourier transform (FT) of its Fe K-edge EXAFS spectra $\left[\kappa^{2}\right.$ 
weighted $\chi(\kappa)]$ exhibited a major peak at $\sim 1.5 \AA$ and a secondary peak(s) at $2-3 \AA$. The former was arising from the backscattering by light atoms $(\mathrm{C}, \mathrm{N}$, or $\mathrm{O})$ in the first coordination shell of the metal center, while the latter was associated with that in the second coordination sphere. The best-fit of the FT-EXAFS spectrum of FeNGR was obtained with four in-plane nitrogen atoms and one or two oxygen atoms as axial ligands (inset of Figure $1 \mathrm{C}$ and Table 1), giving a total coordination number $(\mathrm{CN})$ for FeNGR of 5 or 6 . The average Fe-N and Fe-O bond distances were 1.99 and $2.01 \AA$, respectively. However, it should be noted here that EXAFS alone cannot distinguish between $\mathrm{N}, \mathrm{C}$, and $\mathrm{O}$ atoms. The reasons for choosing four $\mathrm{N}$ atoms as first shell neighbors to $\mathrm{Fe}$ is explained in a previous study from the authors ${ }^{[16]}$. The ${ }^{57} \mathrm{Fe}$ Mössbauer spectrum of FeNGR was fitted with two quadrupole doublets, labelled D1 and D2 (Figure 1D and Table 2), representing 64\% and 36\% of the resonant absorption area, respectively. T. Mineva et al. ${ }^{[17]}$ recently assigned D1 to high spin $\mathrm{Fe}^{3+} \mathrm{N}_{4}-\mathrm{O}$ sites ( $\mathrm{O}$ can be $\mathrm{OH}$ or $\mathrm{O}_{2}$ ) and $\mathrm{D} 2$ to low or medium spin $\mathrm{Fe}^{2+} \mathrm{N}_{4}$ sites, according to combined experimental and theoretical results. In line with the EXAFS and Mössbauer spectra, Fe 2p X-ray photoelectron spectroscopy (XPS) spectrum identifies the presence of both $\mathrm{Fe}^{2+}$ and $\mathrm{Fe}^{3+}$ species in FeNGR (Figure S2A), while nitrogen from $\mathrm{M}-\mathrm{N}_{\mathrm{x}}$ species was also detected by the N1s XPS signal at $\sim 399.5 \mathrm{eV}$ (Figure S2B). In summary, the EXAFS, Mössbauer and XPS results unambiguously demonstrated the local structure of iron in FeNGR as planar Fe-N 4 (possibly with one or two $\mathrm{O}$ in the axial directions), resembling the structure of heme-core.

\section{Abiotic assessment of the enzyme-like activity of FeNGR}

Given that NOX is able to catalyze the conversion of NADPH into NADP ${ }^{+}$along with the production of superoxide, we assessed the enzymatic activity of FeNGR by detection of $\mathrm{NADP}^{+}$and radical generation in phosphate buffered saline (PBS) ${ }^{[18]}$. After $2 \mathrm{~h}$ incubation of NADPH with either FeNGR or pristine GR in air to supply $\mathrm{O}_{2}$, we collected the supernatants of the reaction mixture to examine their UV-Vis spectra and compared them with those recorded for the two standards, NADPH and NADP ${ }^{+}$[19]. As shown in Figure S3, FeNGR could catalyze the conversion of NADPH, evidenced 
by a significant decrease of the absorption peak at $340 \mathrm{~nm}$, proportionally related to NADPH concentration. We further performed high performance liquid chromatography (HPLC) analysis of the FeNGR-catalyzed reaction mixture to specifically identify the products. As shown in Figure 2A, NADP ${ }^{+}$and NADPH standards showed prominent retention peaks at 2.58 and $3.8 \mathrm{~min}$, respectively. Notably, there was no detectable signal that can be assigned to NADPH in the FeNGR-catalyzed reaction mixture, but a significant signal that can be related to the $\mathrm{NADP}^{+}$peak. We then used the integrated area of $\mathrm{NADP}^{+}$peak to estimate the amount of NADPH oxidized into $\mathrm{NADP}^{+}$, and thereby to assess the catalytic efficiency of FeNGR in converting NADPH during this abiotic test. FeNGR showed $92.5 \%$ conversion efficiency of NADPH into NADP ${ }^{+}$, whereas GR had limited effects with only ca $7 \%$ conversion efficiency during the same test (Figure 2A, right panel). The production of superoxide during the FeNGRcatalyzed hydrogen abstraction from NADPH was assessed by 2', 7'-dichloro-dihydrofluorescein $\left(\mathrm{H}_{2} \mathrm{DCF}\right)$. The latter is a non-fluorescent molecule that is converted to the fluorescent 2', 7'-dichlorofluorescein (DCF) in the presence of oxidizing radicals. As shown in Figure 2B, a five-fold increase of DCF fluorescence was detected in the catalysis of NADPH oxidation by the FeNGR materials compared to the fluorescence signal in the catalysis by GR. After that, we evaluated the effect of the reaction conditions (temperature and $\mathrm{pH}$ ) on the catalytic activity. As one can see in Figure $2 \mathrm{C}$, protein oxidase could sustain $>80 \%$ catalytic activity merely in reactions confined at 4-60 ${ }^{\circ} \mathrm{C}$, pH 3-11 and 0-80\% organic solvents. Surprisingly, FeNGR can sustain $>85 \%$ dehydrogenation catalytic activity in more compatible reaction conditions, e.g. temperature up to $90{ }^{\circ} \mathrm{C}$, pH 1-12 and $100 \%$ organic solvents (methanol). These abiotic results indicate that FeNGR can mimic the enzymatic activity of NOX by catalyzing the conversion of NADPH into $\mathrm{NADP}^{+}$along with the generation of oxidizing radicals.

\section{Assessment of enzymatic effects of FeNGR in cells}

While NOX is a transmembrane enzyme and serves as an upstream signaling protein complex to generate superoxide for subsequent immune responses, we then examined 
whether FeNGR could mimic the biological functions of NOX in THP-1 cells. First, we questioned whether FeNGR had similar cellular distribution pattern to NOX. Transmission Electron Microscope (TEM) and confocal laser scanning microscope (CLSM) were exploited to visualize the cellular distribution of FeNGR. As shown in Figure 3A, FeNGR agglomerates with enhanced electron densities were visualized, being attached to the cytoplasmic membranes of THP-1 cells. The latter is a macrophage-like myeloid cell line that often used to study the immune response against stimuli. Energy Dispersive X-ray (EDX) analysis confirmed the presence of Fe element in these agglomerates. To further validate the close association between FeNGR and cell membrane, we resorted to intracellular visualization by CLSM. FeNGR was labeled by fluorescein isothiocyanate (FITC) and cell membranes were labeled by wheat germ agglutinin (WGA) conjugated with Alexa Fluor 594, respectively. As shown in Figure 3B, agglomerates of FITC-labeled FeNGR and the WGA-labeled cell membranes are identified by the green dots (left column) and red circles (central column), respectively. The overlay between the two signals revealed that the FeNGR agglomerates and the cell membrane are closely associated, consistent with the information provided by the TEM images, but with greater detail (Figure 3B). These results indicated that FeNGR had similar cell distributions to NOX, a membrane-bound enzyme, of which cytochrome b558 faces the extracellular space, p47phox, p67phox and G protein Rac are cytosolic subunits.

Next, we assessed the capability of FeNGR to generate superoxide radicals, a key function of NOX towards immunology. THP-1 cells with membranes incorporated by FeNGR were denoted as THP-1 $1^{+\mathrm{FeNGR}}$ and stained by dihydroethidium (DHE) to visualize cellular superoxide resulting from the catalysis of FeNGR. As shown in Figure 4A, compared to untreated THP-1 cells, a massive signal related to superoxide could be detected in THP-1 ${ }^{+ \text {FeNGR }}$ cells, evidenced by the intense red fluorescence. The strong correlation between the presence of FeNGR and the production of superoxide radicals was further demonstrated with knockdown (KD) cells where the expression of gp91phox subunit of NOX is inhibited to reduce superoxide generation. While the 
gp91phox-KD cells failed to generate detectable superoxide, incorporation of FeNGR in cell membranes could retrieve the capability of gp91phox-KD cells to produce superoxide under Alum stimulation. Flow cytometry was used to quantify the percentages of cells displaying DHE-positive staining by superoxide. As shown in Figure 4B (right panel), the incorporation of FeNGR in THP-1 cells significantly increased the superoxide generation in THP-1 cells $(+47 \%)$, while the incorporation of FeNGR in gp91phox-KD cells increased even more this percentage from 30 to $113 \%$.

The produced superoxide radicals may further activate immune signaling pathways in THP-1 cells, to release immune cytokines, such as IL-1 $\beta$, TNF- $\alpha$ and IL-6, which are important mediators of the inflammatory response of macrophages. We therefore detected these cytokines to unambiguously testify the biological function of FeNGR as a nanozyme. As shown in Figure 4C, no cytokine production was detected in gp91phoxKD cells exposed to Alum, whereas incorporation of FeNGR in KD cells induced significant production of IL-1 $\beta$, TNF- $\alpha$ and IL- 6 . This result indicated that deficiency of NOX indeed led to failure of adaptive immune responses to stimuli in THP-1 cells. However, incorporation of FeNGR in gp91phox-KD cells was able to retrieve their immune activity, displaying high level of cytokine production after Alum exposure. Furthermore, we examined the effect of a NOX inhibitor, VAS2870 in THP- $1^{+ \text {FeNGR }}$ cells. As shown in Figure 4D, VAS 2870 could significantly reduce Alum-stimulated IL-1 $\beta$ production in THP-1 cells, whereas it had limited effects in THP- $1^{+ \text {FeNGR }}$ cells. This suggests that the enzymatic activity of FeNGR was not impacted by known inhibitors of the NOX. All these results suggest that FeNGR nano-enzyme is a promising alternative to NOX and could well replicate its biological function in immune activation.

FeNGR belongs to the class of materials usually labelled as M-N-C in the literature (M is typically a $3 \mathrm{~d}$ transition metal), currently investigated intensively for catalyzing oxygen electro-reduction to water in proton exchange membrane fuel cells ${ }^{[20][15]}$ and $\mathrm{CO}_{2}$ electro-reduction to $\mathrm{CO}^{[21]}{ }^{[22]}$. M-N-C materials are prepared by pyrolysis of 
selected metal, nitrogen and carbon precursors, generally resulting in a plurality of active sites from single metal atom sites to metal clusters (metallic particles, metalcarbides, metal oxides, etc). Since 2015 however, controlled synthetic strategies have been established to secure either a) the complete atomic dispersion of the metal atoms, leading solely to $\mathrm{M}-\mathrm{N}_{\mathrm{x}}$ sites and the absence of metal clusters after pyrolysis, as is the case here, or b) to secure the complete aggregation of the metal into metallic or metalcarbide nanoparticles. With such model catalysts, the $M-N_{x}$ sites have been identified to be the most active and selective sites for $\mathrm{O}_{2}$ electro-reduction, especially in acidic medium [15] [23]. In addition, the $\mathrm{MN}_{\mathrm{x}}$ sites have been recently found to display appropriate binding affinity with $\mathrm{H}^{*}$ and are able to assist the abstraction of $\beta$ hydrogen from alcohols ${ }^{[24]}{ }^{[25]}$ and formic acid ${ }^{[26]}$. From this previous knowledge of $\mathrm{MN}_{\mathrm{x}}$ sites' reactivity towards $\mathrm{O}_{2}$ electro-reduction and hydrogen abstraction, a possible mechanism for NAPDH oxidase reactivity of $\mathrm{FeN}_{\mathrm{x}}$ sites is proposed in Scheme 1, involving two steps. First, NADPH is absorbed on FeNGR due to $\pi$ - $\pi$ stacking and the electronegativity of $\mathrm{N}$ atoms of $\mathrm{Fe}-\mathrm{N}_{4}$ sites, acting as basic sites to bind with $\mathrm{H}$ atoms. In parallel, $\mathrm{O}_{2}$ adsorbs on the central iron cation. Second, NADPH donated one-pair of electrons to the Fe-N $\mathrm{N}_{4}$ site, accompanied by the production of $\mathrm{H}^{+}$and $\mathrm{NADP}^{+}$; the electron donation increases the electron density of delocalized $\pi$ bond of FeNRG, resulting in the electron transfer to $\mathrm{O}_{2}$ and superoxide generation.

\section{Conclusions}

In this communication, we report that FeNGR can serve as a nano-enzyme to mimic the enzymatic activity of NOX. The conversion of NADPH to $\mathrm{NADP}^{+}$catalyzed by this nano-enzyme reached $92.5 \%$ along with the generation of superoxide, which is necessary to activate immune signaling pathways. Incorporation of FeNGR in cell membranes retrieved the immune activity of NOX-deficient cells. Overall, this study identifies an alternative nanozyme of NOX, providing new insights for the treatment of NOX related diseases.

\section{Notes}


The authors declare no competing financial interest.

\section{Data and material availability}

The raw data that support the findings of this study are available from the corresponding authors upon request.

\section{Supplementary Materials}

Experimental sections, Structures of heme core and FeNGR, XPS spectra of FeNGR and UV-Vis spectra of FeNGR-treated NADPH.

\section{Acknowledgments}

This work was supported by the National Natural Science Foundation of China (Number 21976126, 31671032), the grants from Key International Cooperation Projects of the Ministry of Science and Technology (2018YFE0120400), Key Project of social development of Jiangsu Province (BE2018653). We thank Dr. Andrea Zitolo (Synchrotron SOLEIL) and Dr. Moulay Tahar Sougrati (ICGM) for measuring and analyzing the XAS and Mössbauer spectra.

\section{Reference}

[1] S. Selemidis, C. G. Sobey, K. Wingler, H. H. Schmidt, G. R. Drummond, Pharmacol Ther 2008, 120, 254-291.

[2] G. R. Drummond, S. Selemidis, K. K. Griendling, C. G. Sobey, Nat Rev Drug Discov $2011,10,453-471$.

[3] S. S. Gregory J Dusting, Fan Jiang, Mem Inst Oswaldo Cruz, Rio de Janeiro 2005, Vol. 100(Suppl. I), 2005.

[4] B. Sun, X. Wang, Z. Ji, M. Wang, Y. P. Liao, C. H. Chang, R. Li, H. Zhang, A. E. Nel, T. Xia, Smal/2015, 11, 2087-2097.

[5] aD. Roos, British Medical Bulletin 2016, 118, 53-66; bS. S. De Ravin, A. Reik, P.-Q. 
Liu, L. Li, X. Wu, L. Su, C. Raley, N. Theobald, U. Choi, A. H. Song, A. Chan, J. R. Pearl, D. E. Paschon, J. Lee, H. Newcombe, S. Koontz, C. Sweeney, D. A. Shivak, K. A. Zarember, M. V. Peshwa, P. D. Gregory, F. D. Urnov, H. L. Malech, Nature Biotechnology 2016, 34, 424-+.

[6] G. J. Gardiner, S. N. Deffit, S. McLetchie, L. Perez, C. C. Walline, J. S. Blum, Frontiers in Immunology 2013, 4.

[7] T. L. L. a. M. GEISZT, ANTIOXIDANTS \& REDOX SIGNALING 2006, Volume 8, Numbers $9 \& 10$.

[8] J. Schuett, H. Schuett, R. Oberoi, A. K. Koch, S. Pretzer, M. Luchtefeld, B. Schieffer, K. Grote, FASEB J 2017, 31, 2612-2624.

[9] S. Yoon, K. Oh, F. Liu, J. H. Seo, G. A. Somorjai, J. H. Lee, K. An, ACS Catalysis 2018, 8, 5391-5398.

[10] Y. Song, K. Qu, C. Zhao, J. Ren, X. Qu, Adv Mater 2010, 22, 2206-2210.

[11] E. Kuah, S. Toh, J. Yee, Q. Ma, Z. Gao, Chemistry 2016, 22, 8404-8430.

[12] aH. Zhang, X. Liang, L. Han, F. Li, Small 2018, 14; bP. Zhang, D. Sun, A. Cho, S. Weon, S. Lee, J. Lee, J. W. Han, D.-P. Kim, W. Choi, Nature Communications 2019, 10.

[13] X. Shen, W. Liu, X. Gao, Z. Lu, X. Wu, X. Gao, Journal of the American Chemical Society 2015, 137, 15882-15891.

[14] M. Gao, Z. Wang, H. Zheng, L. Wang, S. Xu, X. Liu, W. Li, Y. Pan, W. Wang, X. Cai, R. Wu, X. Gao, R. Li, Angew Chem Int Ed Eng/2020, 59, 3618-3623.

[15] A. Zitolo, V. Goellner, V. Armel, M. T. Sougrati, T. Mineva, L. Stievano, E. Fonda, F. 
Jaouen, Nat Mater 2015, 14, 937-942.

[16] J. Li, P. Pršlja, T. Shinagawa, A. J. Martín Fernández, F. Krumeich, K. Artyushkova, P. Atanassov, A. Zitolo, Y. Zhou, R. García-Muelas, N. López, J. Pérez-Ramírez, F. Jaouen, ACS Catalysis 2019, 9, 10426-10439.

[17] T. Mineva, I. Matanovic, P. Atanassov, M.-T. Sougrati, L. Stievano, M. Clémancey, A. Kochem, J.-M. Latour, F. Jaouen, ACS Catalysis 2019, 9, 9359-9371.

[18] S. Kasai, H. Shiku, Y.-s. Torisawa, H. Noda, J. Yoshitake, T. Shiraishi, T. Yasukawa, T. Watanabe, T. Matsue, T. Yoshimura, Analytica Chimica Acta 2005, 549, 14-19.

[19] K. Shah, T. Imae, Journal of Materials Chemistry A 2017, 5, 10.1039.C1037TA01861D.

[20] M. Lefevre, E. Proietti, F. Jaouen, J. P. Dodelet, Science 2009, 324, 71-74.

[21] L. Li, B. Miao, Z. Li, Z. Sun, N. Peng, ACS Sens 2019, 4, 2738-2745.

[22] A. S. Varela, W. Ju, A. Bagger, P. Franco, J. Rossmeisl, P. Strasser, ACS Catalysis $2019,9,7270-7284$.

[23] A. Zitolo, N. Ranjbar-Sahraie, T. Mineva, J. Li, Q. Jia, S. Stamatin, G. F. Harrington, S. M. Lyth, P. Krtil, S. Mukerjee, E. Fonda, F. Jaouen, Nat Commun 2017, 8, 957.

[24] L. Zhang, A. Wang, W. Wang, Y. Huang, X. Liu, S. Miao, J. Liu, T. Zhang, ACS Catalysis 2015, 5, 6563-6572.

[25] J. Xie, K. Yin, A. Serov, K. Artyushkova, H. N. Pham, X. Sang, R. R. Unocic, P. Atanassov, A. K. Datye, R. J. Davis, ChemSusChem 2017, 10, 359-362.

[26] Y. Leng, S. Du, G. Feng, X. Sang, P. Jiang, H. Li, D. Wang, ACS App/ Mater Interfaces $2020,12,474-483$. 

Tables

Table 1

Fitting results of EXAFS

\begin{tabular}{ll}
\hline & FeNGR \\
\hline $\operatorname{RM-N}(\AA)$ & $1.99(2) / 2.01(2)$ \\
$\sigma^{2}\left(\AA^{2}\right)$ & $0.008(2)$ \\
$\mathbf{C N}$ & $5 / 6$ \\
\hline
\end{tabular}

Table 2

Fitting results of the ${ }^{57} \mathrm{Fe}$ Mössbauer spectrum of FeNGR

\begin{tabular}{cccccc}
\hline Comp. & RA (\%) & IS $\left(\mathbf{m m} \cdot \mathbf{s}^{-1}\right)$ & QS $\left(\mathbf{m m} \cdot \mathbf{s}^{-1}\right)$ & $\mathbf{L W ~}\left(\mathbf{m m} \cdot \mathbf{s}^{-1}\right)$ & Assignment \\
\hline D1 & 64 & 0.34 & 0.94 & 0.65 & ${\text { High spin } \mathrm{Fe}^{3+} \mathrm{N}_{4}-\mathrm{O}}$ \\
D2 & 36 & 0.36 & 2.59 & 1.42 & Low spin $\mathrm{Fe}^{2+} \mathrm{N}_{4}$ \\
\hline
\end{tabular}


Figure Legend:

Figure 1 Characterization of the physicochemical properties of GR and FeNGR samples

A) AFM, B) Raman, C) FT-EXAFS and D) ${ }^{57} \mathrm{Fe}$ Mössbauer spectra of Fe-N doped Graphene. A drop of GR and FeNGR suspension $(50 \mu \mathrm{g} / \mathrm{mL})$ was placed on a mica plate, and then dried at room temperature for AFM observation (scale bar, $500 \mathrm{~nm}$ ). FeNGR or GR powder were used for Raman, EXAFS and Mössbauer measurements.

Figure 2 Abiotic assessment of the enzymatic activity of FeNGR

A) LC analysis of the reaction products of NADPH dehydrogenation catalyzed by FeNGR. NADPH was reacted with $100 \mu \mathrm{g} / \mathrm{ml}$ FeNGR or GR for $2 \mathrm{~h}$ in air. The products, NADPH and $\mathrm{NADP}^{+}$standards were separated by LC with UV-Vis detection. B) Assessment of oxygen radical formation by DCF assay. FeNGR or GR at $50 \mu \mathrm{g} / \mathrm{ml}$ were mixed with $100 \mu \mathrm{g} / \mathrm{ml}$ NADPH in $10 \mu \mathrm{L}$ PBS solution of 96-well plate, followed by addition of $90 \mu \mathrm{L}$ DCF working solutions. The generation of radicals was examined by the fluorescence at $520 \mathrm{~nm}$. C) Effects of temperature, $\mathrm{pH}$ and organic solvent on catalytic activity of FeNGR and peroxidase. FeNGR and peroxidase at $200 \mu \mathrm{g} / \mathrm{mL}$ were pretreated in DI $\mathrm{H}_{2} \mathrm{O}$ at $4-90{ }^{\circ} \mathrm{C}, 10 \mathrm{mM}$ phosphate solutions at $\mathrm{pH}$ 1-12 or different ratios of $\mathrm{MeOH} / \mathrm{H}_{2} \mathrm{O}$ solutions $(0 \%, 20 \%, 40 \%, 60 \%, 80 \%, 100 \%)$ for $2 \mathrm{~h}$. The activities of the treated catalysts were assessed by DCF assay.

\section{Figure 3 Visualization of the cellular distribution of FeNGR}

A) TEM images showing the interaction between FeNGR and THP-1 cells. THP-1 cells treated by $25 \mu \mathrm{g} / \mathrm{mL}$ FeNGR for $6 \mathrm{~h}$ were collected to fix, stain and prepare slides for TEM observation. Asterisks in the EDX spectra represent background elements (scale bar, $5 \mu \mathrm{m}$ and $1 \mu \mathrm{m}$ ). B) Confocal imaging of FeNGR distribution in cells. THP-1 cells treated by $25 \mu \mathrm{g} / \mathrm{mL}$ FITC-labeled FeNGR for $6 \mathrm{~h}$, were washed twice by PBS. Membranes and nuclei were stained by WGA conjugated with Alexa Fluor 594 (red) 
and Hoechst 33342 (blue), respectively.

Figure 4 Assessments of the biological functions of FeNGR as nanozyme of NOX A) Confocal imaging and B) flow cytometry analysis of superoxide generation in cells. THP- $1^{+\mathrm{FeNGR}}$ and KD-THP- $1^{+\mathrm{FeNGR}}$ cells were acquired by incubation of THP-1 and KD-THP-1 cells with $25 \mu \mathrm{g} / \mathrm{mL}$ FeNGR for $6 \mathrm{~h}$, respectively. Then the cells were exposed to $25 \mu \mathrm{g} / \mathrm{mL}$ Alum for $24 \mathrm{~h}$, followed by DHE and Hoechst 33342 staining for confocal microscopy and flow cytometry analysis. Percentages of superoxide generation were calculated with the following equation: $\%$ Superoxide generation $=\frac{F_{t}}{F_{c}} \times 100 \%$, where Fc and Ft represent the fluorescence intensity DHE in THP-1 cells and treated THP-1 cells (by FeNGR or shRNA), respectively. The scale bars of confocal images are $5 \mu \mathrm{m} .{ }^{* * *} \mathrm{p}<0.001$ compared to THP-1 cells, \#\#\#p<0.001 compared to KD-THP-1 cells by two tailored Student T-test. C) Comparison of Cytokine release in KD-THP-1 and KD-THP- $1^{+\mathrm{FeNGR}}$. Both cell lines were exposed to $25 \mu \mathrm{g} / \mathrm{mL}$ Alum for $24 \mathrm{~h}$. TNF- $\alpha$, IL-1 $\beta$ and IL-6 in supernatants were measured by ELISA. ${ }^{* *} \mathrm{p}<0.001$ compared to THP-1 cells by two tailored Student Ttest. D) Impacts of NOX inhibitors on FeNGR nanozyme. THP-1 and KD-THP-1 ${ }^{+\mathrm{FeNGR}}$ cells were pretreated with $10 \mathrm{mM}$ VAS2870 $(10 \mathrm{mmol} / \mathrm{L})$ for $1 \mathrm{~h}$ before exposure to 25 $\mu \mathrm{g} / \mathrm{mL}$ Alum for $6 \mathrm{~h} .{ }^{*} \mathrm{p}<0.05$ compared to THP-1 cells without VAS2870 treatment by two tailored Student T-test.

\section{Scheme 1 Proposed oxidation reaction steps of NADPH catalyzed by FeNGR}

First, NADPH is absorbed on FeNGR via $\pi-\pi$ stacking and the electronegativity of N atoms involved in the $\mathrm{Fe}-\mathrm{N}_{4}$ sites. In parallel, the central iron cation adsorbs $\mathrm{O}_{2}$. Second, NADPH donates one-pair of electrons to the $\mathrm{Fe}_{-} \mathrm{N}_{4}$ site for hydrogen abstraction as well as electron transfer to $\mathrm{O}_{2}$ for superoxide generation. 
Figure 1

A
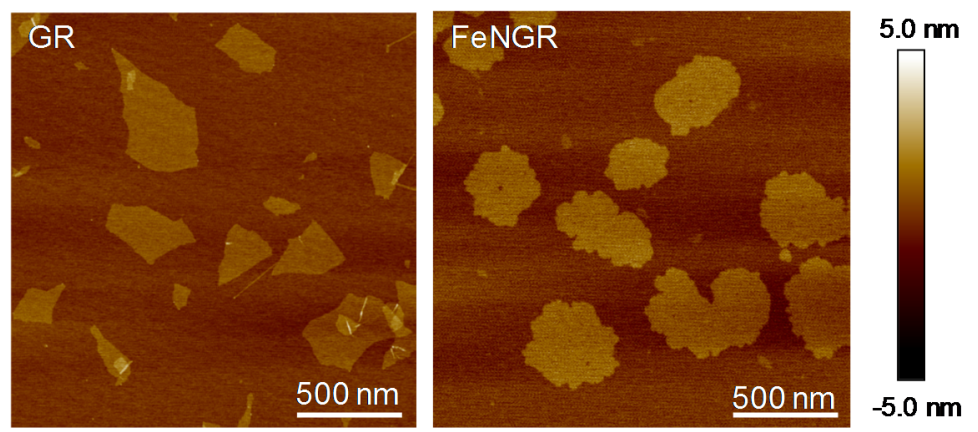

B
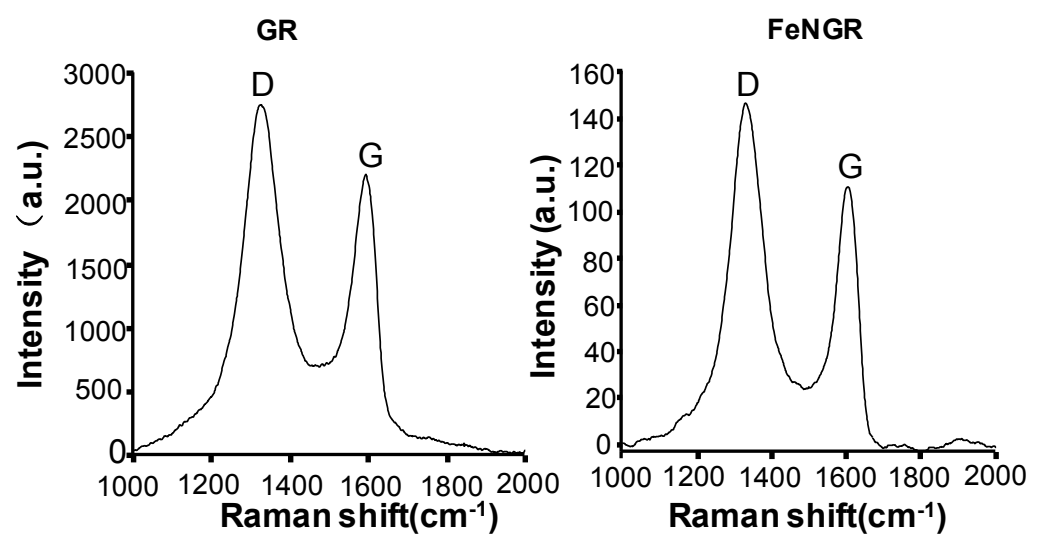

C

D
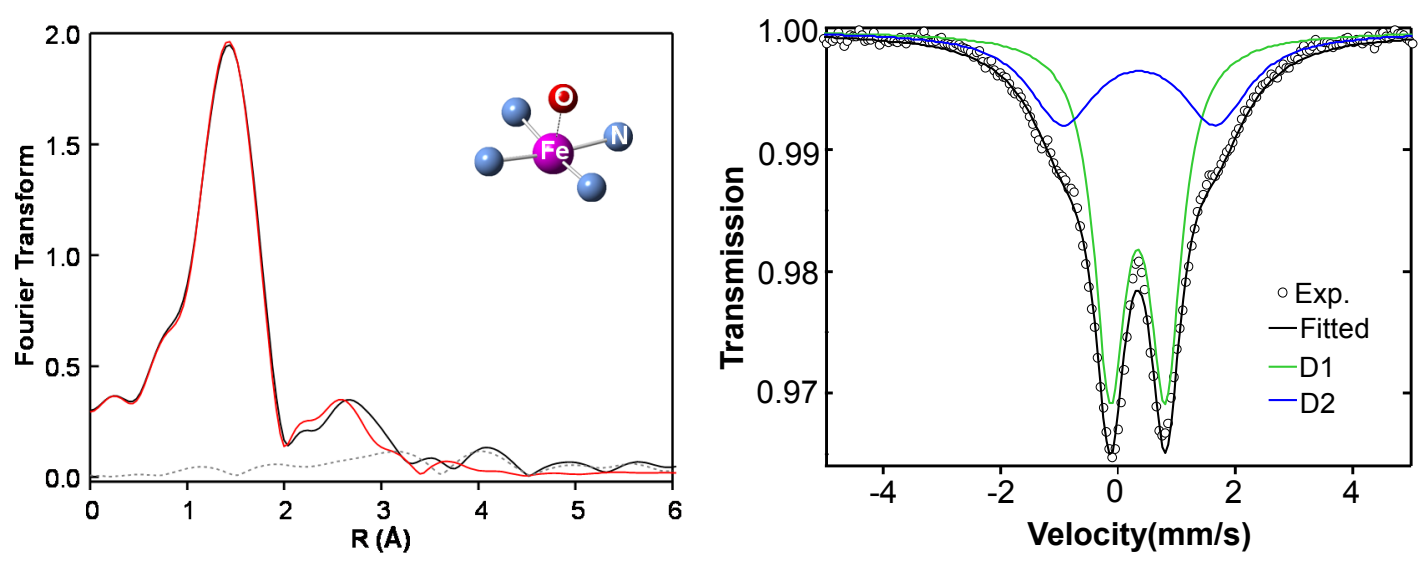
Figure 2

A
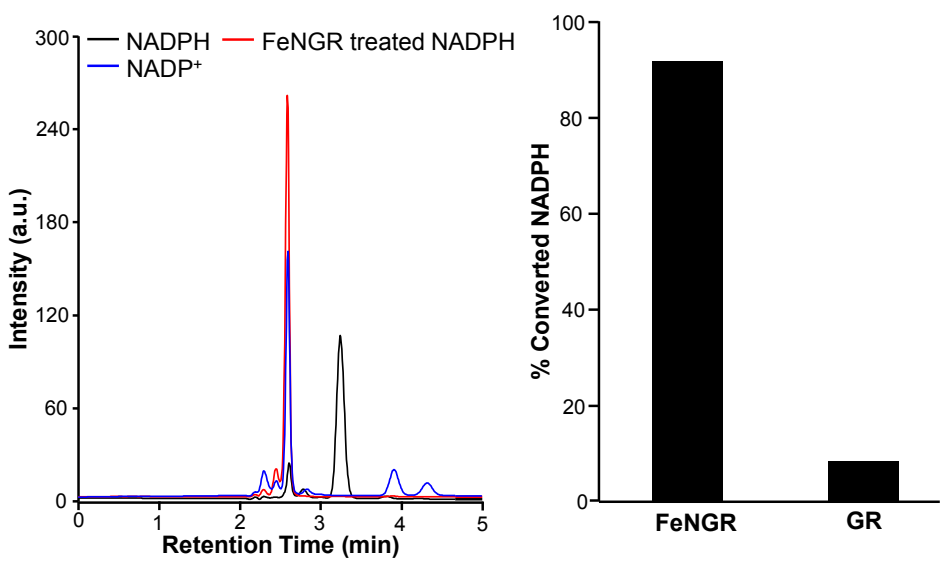

B
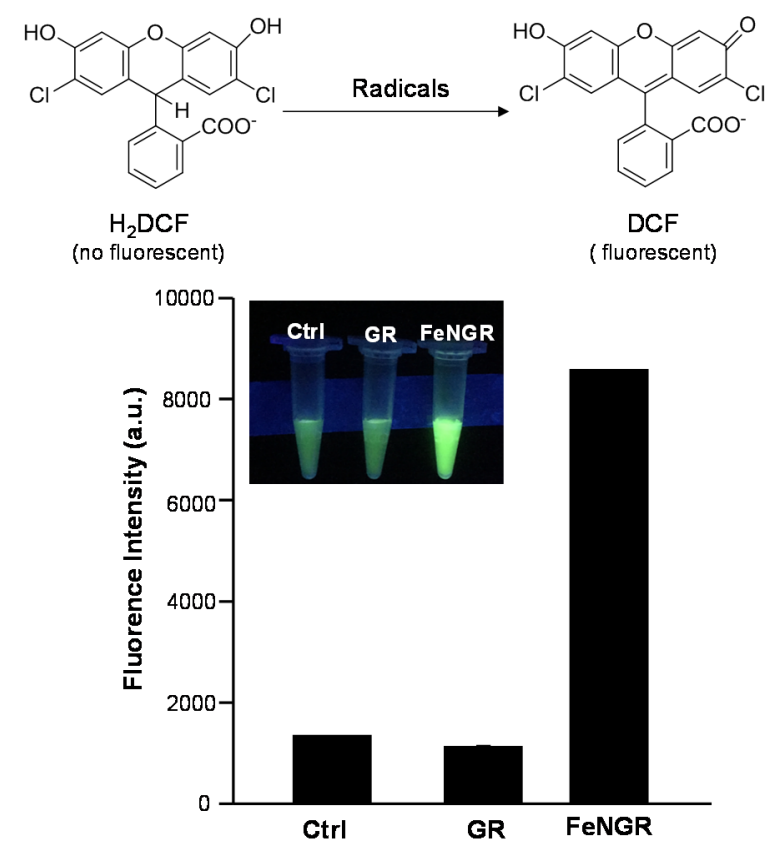

C
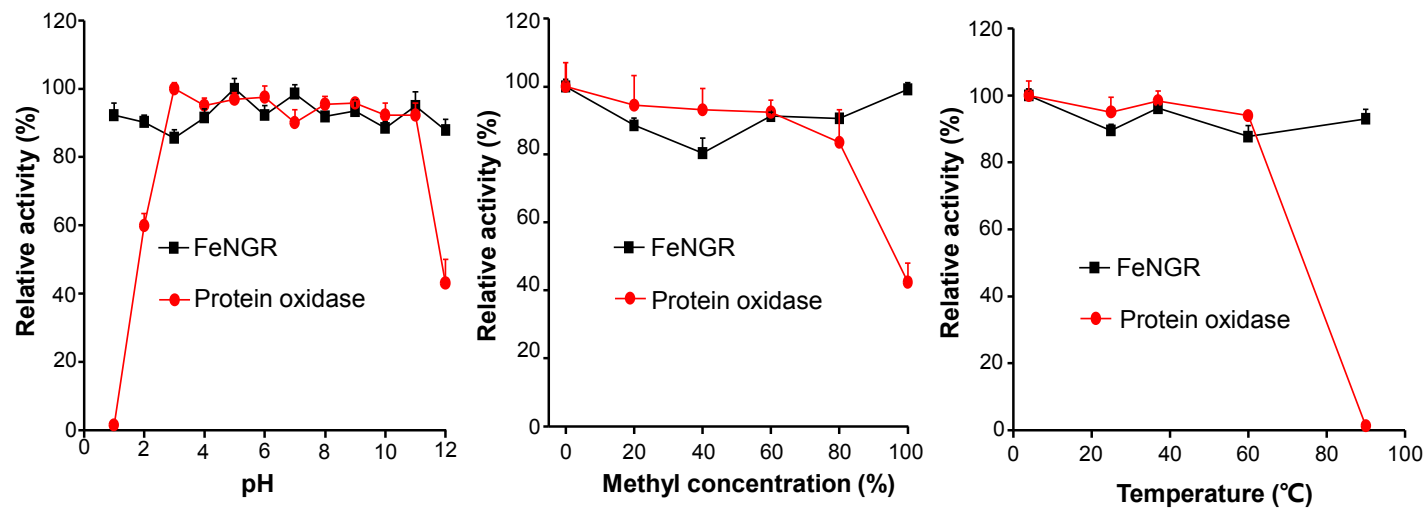
Figure 3

A

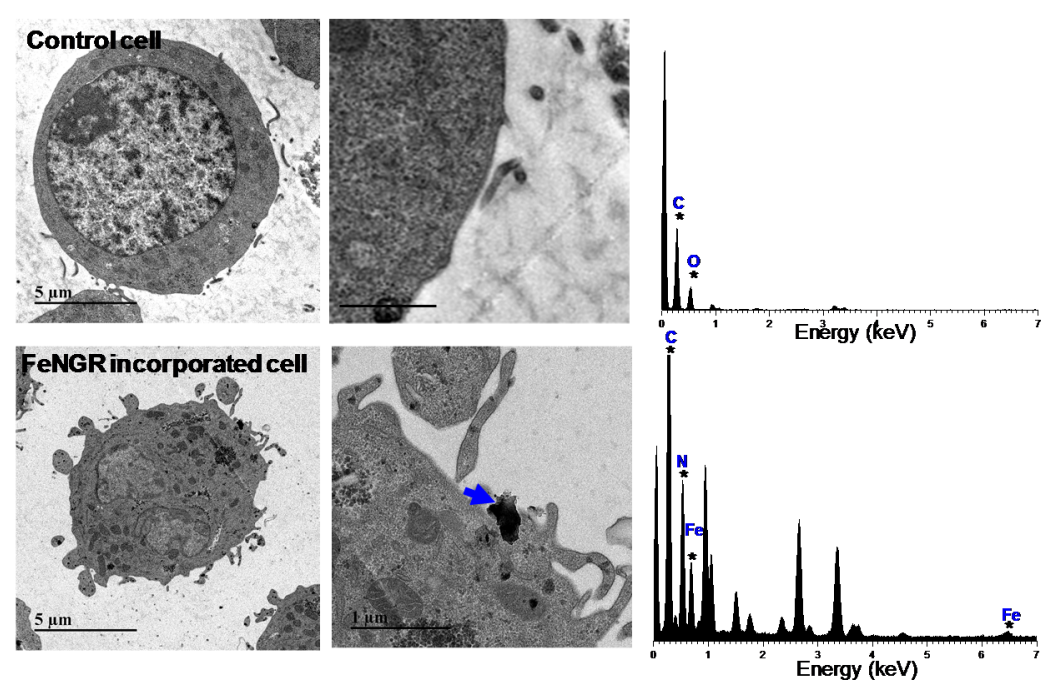

B

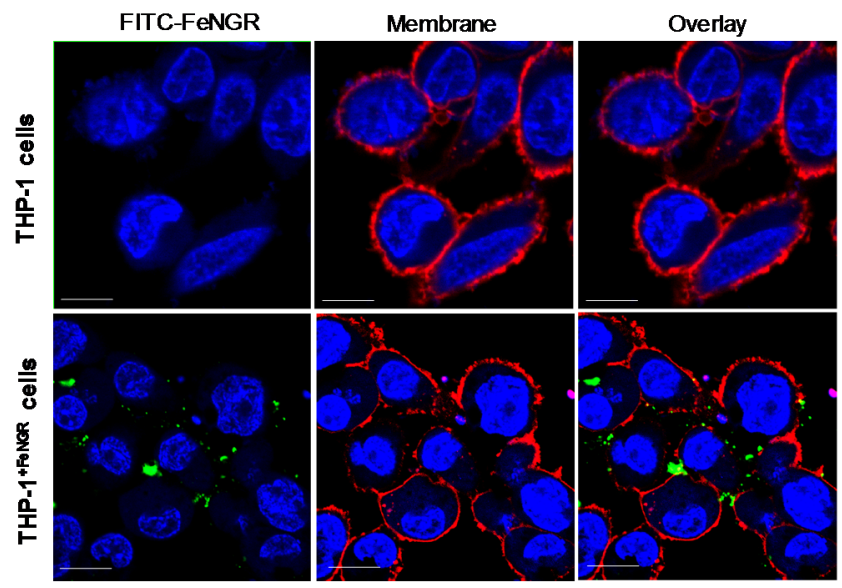


Figure 4

A

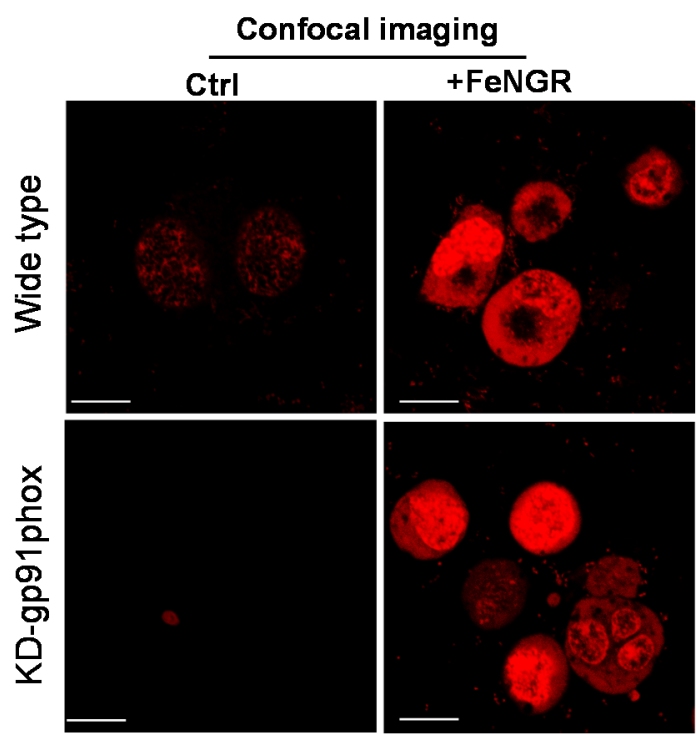

C

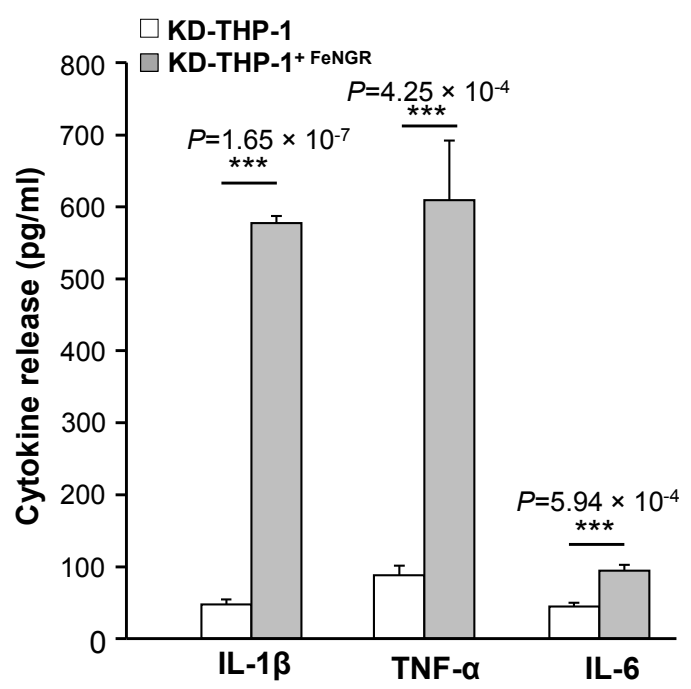

B

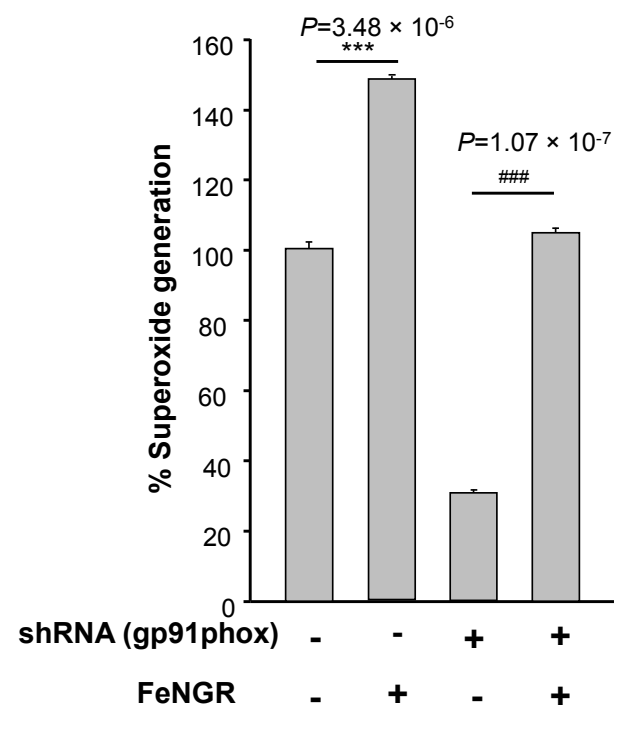

D

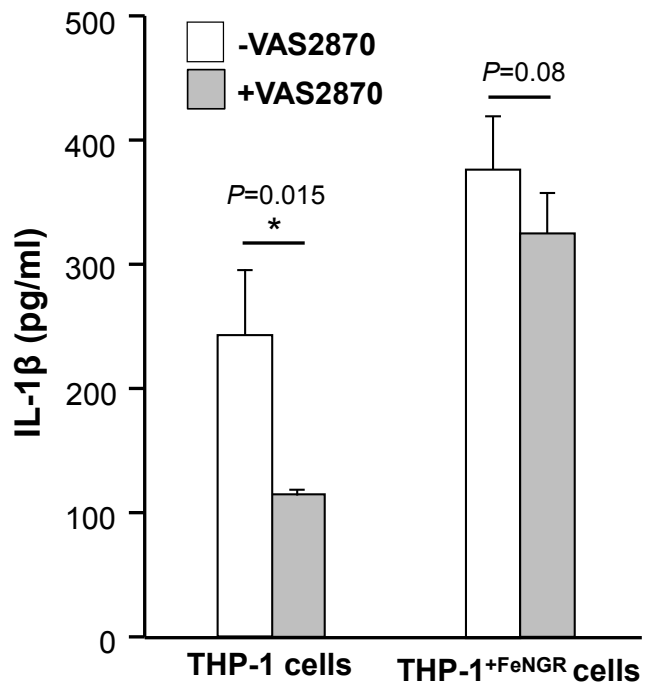


Scheme 1

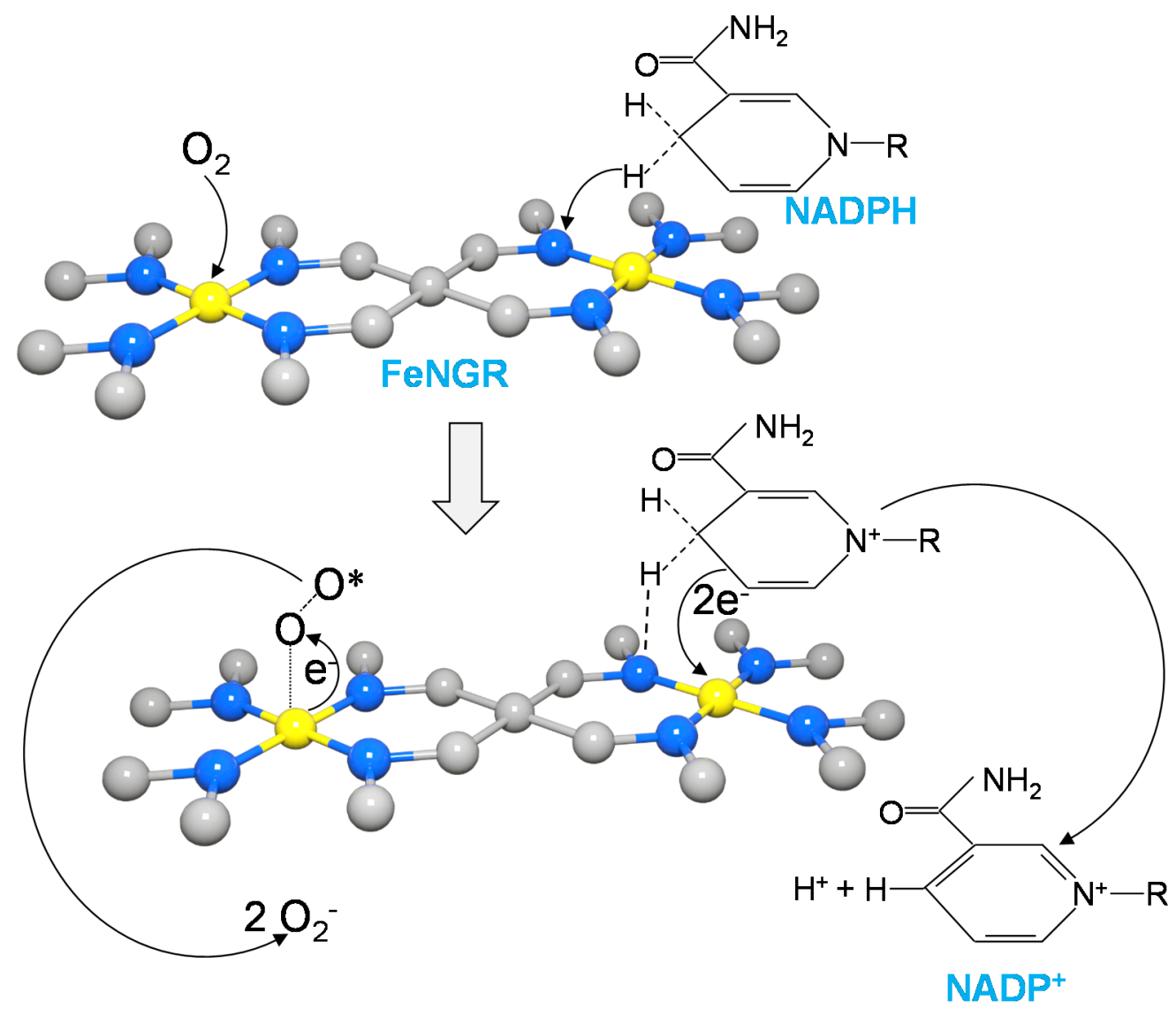

